\title{
Identification of key genes and pathways associated with obesity in children
}

\author{
LING LI $^{1 *}$, GUANGYU WANG ${ }^{1 *}$, NING LI $^{1}$, HAIYAN YU ${ }^{2}$, JIANPING SI ${ }^{3}$ and JIWEN WANG ${ }^{4-6}$ \\ ${ }^{1}$ Department of Paediatrics, Jinan Children's Hospital; ${ }^{2}$ Department of Paediatrics, The Fifth People's Hospital of Jinan, \\ Jinan, Shandong 250022; ${ }^{3}$ Department of Pediatrics, The People's Hospital of Guangrao, Dongying, Shandong \\ 257300; ${ }^{4}$ Department of Neurology, Children's Medical Center, Qilu Hospital of Shandong University; ${ }^{5}$ Brain \\ Science Research Institute, Shandong University, Jinan, Shandong 250012; ${ }^{6}$ Department of Neurology, Shanghai \\ Children's Medical Center, Shanghai Jiaotong University School of Medicine, Shanghai 200240, P.R. China
}

Received March 16, 2016; Accepted February 24, 2017

DOI: 10.3892/etm.2017.4597

\begin{abstract}
The present study aimed to identify potential key genes and pathways in obese children in order to explore possible molecular mechanisms associated with child obesity. The array dataset GSE29718 was downloaded from the Gene Expression Omnibus database. Subcutaneous adipose tissue samples derived from 7 obese children and 8 lean children were selected for the analysis. Differentially expressed genes (DEGs) in samples from obese children compared with those from lean children were analyzed by the limma package. Gene ontology (GO) annotation, Kyoto Encyclopedia of Genes and Genomes and Reactome pathway enrichment analyses for up and downregulated genes were performed. A protein-protein interaction (PPI) network was constructed with Cytoscape software and important genes associated with obesity were determined using IRegulon. A total of 199 DEGs (79 up and 120 downregulated genes) were identified in the samples of obese children compared with those from lean children. The PPI network was established with 103 nodes and 147 protein pairs. Matrix metalloproteinase 9 (MMP9) and acetyl-CoA carboxylase $\beta$ (ACACB) were identified as hub genes in the PPI network and may therefore be marker genes for child obesity. In addition, upregulated DEGs were enriched in Reactome pathways associated with the immune system. Besides, MMP9 was upregulated in immune system processes as a GO term in the category Biological Processes. The results of the present
\end{abstract}

Correspondence to: Dr Jiwen Wang, Department of Neurology, Shanghai Children's Medical Center, Shanghai Jiaotong University School of Medicine, 1678 Dongfang Road, New Pudong, Shanghai 200240, P.R. China

E-mail: wjiwen57@sina.com

\section{${ }^{*}$ Contributed equally}

Key words: child obesity, differentially expressed genes, The Kyoto Encyclopedia of Genes and Genomes pathway, reactome pathway, protein-protein interaction network study indicated that MMP9, ACACB and immune system pathways may have a significant role in child obesity.

\section{Introduction}

Obesity is a medical condition in which excess body fat has accumulated to a certain degree, and affected individuals may have a reduced life expectancy and increased health problems (1). Child obesity is becoming a health problem in developed and developing countries $(2,3)$. Obesity is caused by a combination of genetic, behavioral, social, cultural, metabolic and physiological factors (4). Child obesity increases the likelihood of certain diseases, such as hyperlipidemia, insulin resistance and hypertension (5). The physiological mechanisms associated with obesity have been investigated in-depth (6), but certain key molecular mechanisms involved in obesity have remained to be identified.

Previous studies have determined certain pathological mechanisms associated with obesity. One study indicated that body fat and weight were inversely associated with 25-hydroxyvitamin $\mathrm{D}$ levels and volumetric dilution may explain for the low vitamin D levels in obese individuals (7). It also has been suggested that a high-fat diet accelerated obesity via the Toll-like receptor 4 signaling pathway (8). In addition, leptin-melanocortin signaling is a key pathway in regulating food intake and body weight, and mutation of ligands or receptors in this pathway may cause obesity (9). Iodothyronine deiodinase type 2 has a role in the progression of obesity via the c-Jun N-terminal kinase (JNK) signaling pathway (10). Activation of the adenosine monophosphate-activated protein kinase signaling pathway has been reported to prevent obesity (11). Furthermore, Vohl et al (12) reported that 216 and 131 genes were overexpressed in visceral fat and subcutaneous adipose tissues, respectively. Linder et al (13) reported that several genes, including the phospholipid transfer gene, ras, adipsin and calcyclin were differentially expressed in adipose tissue of male or female patients. In addition, two previous studies indicated that variation in the fat mass and obesity-associated gene was associated with childhood obesity and severe adult obesity $(14,15)$. However, to the best of our knowledge, few articles have reported the genes or pathways of 
childhood obesity alone. Therefore, further significant genes or pathways associated with child obesity are required to be identified.

The microarray data of GSE29718 have been previously used to reveal the links between type 2 diabetes and obesity (16) or used to detect molecular mechanisms for the association between obesity and colorectal cancer (17). The present study identified the differentially expressed genes (DEGs) in obese children compared with those in lean children based on the microarray data of GSE29718. Functional enrichment analyses of DEGs were then performed. In addition, a protein-protein interaction (PPI) network was established and important genes associated with obesity were analyzed. The present study aimed to identify critical genes or pathways associated with child obesity and explored possible underlying molecular mechanisms.

\section{Materials and methods}

Affymetrix microarray data. The array dataset GSE29718 deposited by Tam et al (18) was downloaded from the Gene Expression Omnibus database (http://www.ncbi.nlm.nih. gov/geo/). The dataset contained 5 visceral adipose tissue samples and 15 subcutaneous adipose tissue samples from children. Only the subcutaneous adipose tissue samples derived from 7 obese and 8 lean children were used for the subsequently analysis. The array data were based on the GPL6244 Affymetrix Human Gene 1.0 ST Array platform, transcript (gene) version (Affymetrix Inc., Santa Clara, CA, USA).

Data pre-processing. The raw data were pre-processed by the robust multiarray average (19) algorithm with the use of oligo (20) in the R software of Bioconductor (Seattle, Washington). The process of pre-processing included background correction, normalization and calculation of gene expression. Finally, a total of 18,977 gene expression values was obtained.

DEG analysis. The DEGs in samples from obese children compared with those from lean children were analyzed by the limma package (21) in Bioconductor. The P-values of the DEGs were calculated by using the unpaired Student's t-test (22) in the limma package in the process of analysis. $\log _{2} \mathrm{FCl} \geq 0.4$ and $\mathrm{P}<0.05$ were set as cut-off criteria.

Gene ontology (GO) and pathway enrichment analyses. GO includes 3 categories, namely molecular function, biological processes (BP) and cellular components, and is a tool used for gene annotation by using a defined, structured and controlled vocabulary (23). The Kyoto Encyclopedia of Genes and Genomes (KEGG) is a database used to assign sets of DEGs to specific pathways (24). Reactome is a database used for forging a link between metabolome and genome (25).

Up and downregulated genes were subjected to GO annotation as well as KEGG and Reactome pathway enrichment analyses. $\mathrm{P}<0.05$ was used as the threshold value, and the number of genes enriched in each pathway was $\geq 2$.

PPI network analysis. The Search Tool for the Retrieval of Interacting Genes (STRING) (26) can be used to provide information regarding predicted and experimental interactions of proteins and the prediction method of this database is from neighborhood, gene fusion, co-occurrence, co-expression experiments, databases and textmining. The DEGs were mapped into PPIs and a combined score of $>0.4$ was set as a threshold value in this study. PPI networks were constructed with Cytoscape software (27). Moreover, the nodes with higher degrees of interaction were considered as hub nodes.

The association among nodes with a higher degree of interaction in the network and the associated biological functions were analyzed by literature mining. GenCLiP 2.0 (28) (http://ci.smu.edu.cn/GenCLiP2.0/confirm_keywords.php) is an online tool used for the literature mining analysis of human genes and networks. Hub gene sets obtained from the PPI network were used as input gene sets. Based on the user-defined query terms, the Literature Mining Gene Networks module can construct gene networks, generate sub-networks and calculate the likelihood of the networks' random occurrence using random simulation (29). In order to annotate the input genes, Gene Cluster with Literature Profiles module can generate statistically over-represented keywords. The keywords grouped by a fuzzy algorithm can be provided by the user or are generated according to occurrence of free terms in the literature on the respective gene. The relevant Medline abstracts (co-occurrence of genes and keywords are highlighted) are linked via the associations between genes and keywords. The co-citation network of key genes in the literature was analyzed using the 'Literature Mining Gene Networks' module in GenCLiP to analyze hot associated genes in a literature research. Subsequently, the associated biological function of these hot genes by 'Gene Cluster with Literature Profiles' modules with $\mathrm{P} \leq 1 \times 10^{-6}$ and hit $\geq 6$.

Analyses of important genes and transcription factors associated with obesity. The comparative toxicogenomics database (CTD) (30) is a tool used to formalize, harmonize and centralize data of genes and proteins across different species. The present study assessed whether any of the marker genes among the DEGs identified had been previously listed as markers of obesity in the CTD database. 'Obesity' was used as an input key word in CTD. Subsequently, the cytoscape plugin iRegulon (31) was used to analyze transcription factors regulating marker genes. iRegulon uses cis-regulatory sequence analysis to reverse-engineer the transcriptional regulatory network underlying a co-expressed gene set. It integrates the transcription factor information from databases such as Transfac, Jaspar, Encode, Swissregulon and Homer, and detects enriched transcription factor motifs and optimal sets of their direct targets by means of genome-wide ranking and recovery. Parameter settings were as follows: Minimum identity between orthologous genes $=0.05$ and maximum false discovery rate on motif similarity $=0.001$. The Normalized Enrichment Score (NES) was the output result. The higher the scores were, the more reliable the results were. Transcription factors and target gene pairs with NES $>5$ were selected.

\section{Results}

DEG analysis. As shown in Fig. 1, a total of 199 DEGs (79 up and 120 downregulated genes) were identified in samples from 

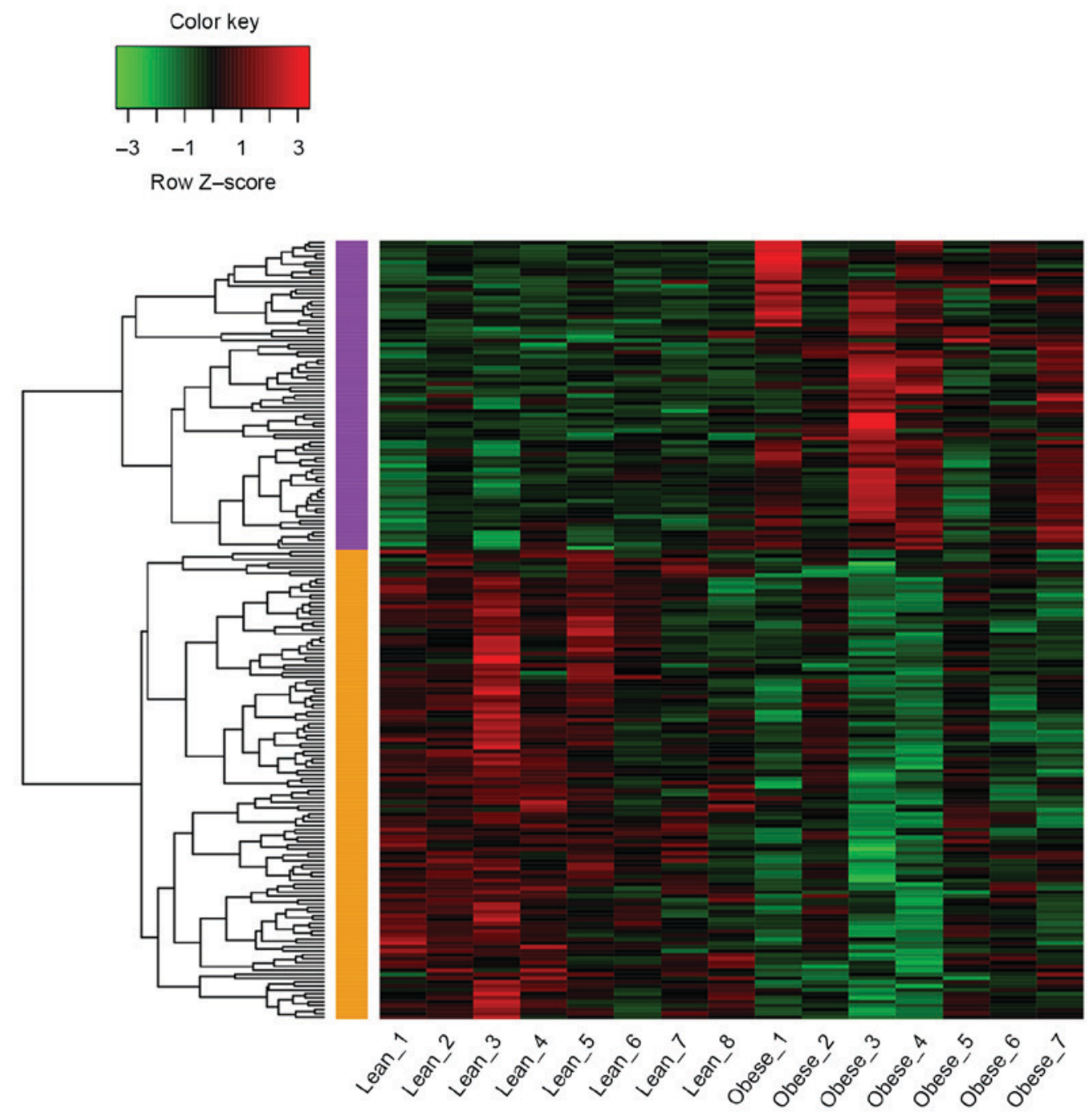

Figure 1. Heat map of differentially expressed genes. Green colour represents a lower and red colour represents a higher expression level. Black colour indicates no differential expression.

obese children compared with those from lean children with $\mathrm{P}<0.05$ and $\mid \log _{2} \mathrm{FCl} \geq 0.4$. The average $\log _{2} \mathrm{FC}$ of upregulated genes was 0.585 and that of downregulated genes was -0.558 .

GO and pathway enrichment analyses. GO, KEGG pathway and Reactome pathway analyses were performed for up and downregulated DEGs. Upregulated DEGs were mainly enriched in the GO terms of extracellular space, immune response and immune system process (Table I). In addition, matrix metalloproteinases 9 (MMP9) was significantly enriched in pathways of immune system processes. The downregulated DEGs were mainly associated with the regulation of system process and cyclic guanosine monophosphate (cGMP)-inhibited cyclic nucleotide (Table I).

The significantly enriched KEGG pathways of upregulated DEGs were cell adhesion molecules and phagosome (Table IIA). The significantly enriched KEGG pathways of downregulated DEGs were nitrogen metabolism and propanoate (Table IIA). The significantly enriched Reactome pathways of upregulated DEGs were immune system and adaptive immune system (Table IIB). The significantly enriched Reactome pathways by downregulated DEGs were signaling by retinoic acid and cGMP effects (Table IIB).
PPI network analysis. A total of 103 nodes and 147 protein pairs were obtained with a PPI score of $>0.4$ based on the STRING database (Fig. 2). The interactions of proteins encoded by the DEGs were inconspicuous. The proteins that were closely associated with other proteins with a degree of interaction $\geq 10$ were MMP9 (degree=16), Acetyl-CoA carboxylase $\beta$ (ACACB; degree=13), MET proto-oncogene, receptor tyrosine dinase (MET; degree=11) and von Willebrand factor (VWF; degree $=10$ ). Literature mining was performed for 8 genes with high degree of interaction in the networks. The co-citation network of these 8 genes in the reported literature is shown in Fig. 3A and the results reported by previous studies regarding these genes are shown in Table III. Significant results of enrichment analysis of these 8 genes reported the literature are shown in Fig. 3B, and enrichment was identified in terms such as binding protein 1 , reactive oxygen species and kinase activity.

Analysis of marker genes and transcription factors associated with obesity. A total of 11 previously reported marker genes of obesity listed in the CTD, including ACACB, MMP9, glutamate-ammonia ligase and ferritin light polypeptide, were identified among the DEGs of the present study. The 
Table I. GO analysis of differentially expressed genes $(\mathrm{P}<0.05)$.

\begin{tabular}{|c|c|c|c|}
\hline GO ID & Term & No. of genes & P-value \\
\hline \multicolumn{4}{|c|}{ Upregulated genes } \\
\hline \multicolumn{4}{|c|}{ GO-BP terms } \\
\hline GO:0006955 & Immune response & 25 & $2.25 \times 10^{-10}$ \\
\hline GO:0002376 & Immune system process & 31 & $6.67 \times 10^{-10}$ \\
\hline GO:0006954 & Inflammatory response & 15 & $1.57 \times 10^{-8}$ \\
\hline GO:0009605 & Response to external stimulus & 27 & $2.20 \times 10^{-8}$ \\
\hline GO:0006952 & Defense response & 23 & $2.41 \times 10^{-8}$ \\
\hline \multicolumn{4}{|l|}{ GO-CC terms } \\
\hline GO:0005615 & Extracellular space & 21 & $1.26 \times 10^{-8}$ \\
\hline GO:0005576 & Extracellular region & 36 & $4.13 \times 10^{-6}$ \\
\hline GO:0044421 & Extracellular region part & 32 & $5.06 \times 10^{-6}$ \\
\hline GO:0005578 & Proteinaceous extracellular matrix & 7 & $4.62 \times 10^{-4}$ \\
\hline GO:0005886 & Plasma membrane & 32 & $9.04 \times 10^{-4}$ \\
\hline \multicolumn{4}{|l|}{ GO-MF terms } \\
\hline GO:0043394 & Proteoglycan binding & 3 & $1.83 \times 10^{-4}$ \\
\hline GO:0001948 & Glycoprotein binding & 4 & $3.47 \times 10^{-4}$ \\
\hline GO:0008061 & Chitin binding & 2 & $4.62 \times 10^{-4}$ \\
\hline GO:0043395 & Heparan sulfate proteoglycan binding & 2 & $1.94 \times 10^{-3}$ \\
\hline GO:0030246 & Carbohydrate binding & 5 & $5.04 \times 10^{-3}$ \\
\hline \multicolumn{4}{|c|}{ Downregulated genes } \\
\hline \multicolumn{4}{|c|}{ GO-BP terms } \\
\hline GO:0044057 & Regulation of system process & 16 & $4.50 \times 10^{-9}$ \\
\hline GO:0008015 & Blood circulation & 15 & $3.72 \times 10^{-8}$ \\
\hline GO:0003013 & Circulatory system process & 15 & $3.97 \times 10^{-8}$ \\
\hline GO:0051239 & Regulation of multicellular organismal process & 36 & $5.25 \times 10^{-8}$ \\
\hline GO:2000021 & Regulation of ion homeostasis & 10 & $1.20 \times 10^{-7}$ \\
\hline \multicolumn{4}{|l|}{ GO-CC terms } \\
\hline GO:0031093 & Platelet alpha granule lumen & 4 & $1.00 \times 10^{-4}$ \\
\hline GO:0031091 & Platelet alpha granule & 4 & $6.00 \times 10^{-4}$ \\
\hline GO:0034774 & Secretory granule lumen & 4 & $6.00 \times 10^{-4}$ \\
\hline GO:0009925 & Basal plasma membrane & 3 & $7.00 \times 10^{-4}$ \\
\hline GO:0060205 & Cytoplasmic membrane-bounded vesicle lumen & 4 & $1.40 \times 10^{-3}$ \\
\hline \multicolumn{4}{|l|}{ GO-MF terms } \\
\hline GO:0004119 & cGMP-inhibited cyclic nucleotide phosphodiesterase activity & 2 & $3.65 \times 10^{-5}$ \\
\hline GO:0043168 & Anion binding & 31 & $1.00 \times 10^{-4}$ \\
\hline GO:0004740 & Pyruvate dehydrogenase (acetyl-transferring) kinase activity & 2 & $2.00 \times 10^{-4}$ \\
\hline GO:0004114 & $3^{1}, 5^{\prime}-$ Cyclic nucleotide phosphodiesterase activity & 3 & $4.00 \times 10^{-4}$ \\
\hline GO:0097367 & Carbohydrate derivative binding & 26 & $4.00 \times 10^{-4}$ \\
\hline
\end{tabular}

BP, biological process; CC, cellular component; MF, molecular function; GO, gene ontology; cGMP, cyclic guanosine monophosphate.

expression levels of these 11 genes in the samples from obese and lean children are shown in Fig. 4A.

The transcription regulatory network of these marker genes is shown in Fig. 4B. The transcription factors with an NES score $>5$ were EP300 (E1A Binding Protein P300, $\mathrm{NES}=6.63$ ), FOXA3 (Forkhead Box A3, NES=6.286), SRY (Sex Determining Region Y, NES=6.11), SNAI2 (NES=5.925), SRF (Serum Response Factor, NES=5.64), MAFA (MAF BZIP Transcription Factor A, NES=5.464), GCM1 (Glial
Cells Missing Homolog 1, NES=5.157) and VDR (Vitamin D (1,25-Dihydroxyvitamin D3) Receptor, NES=5.115).

\section{Discussion}

In the present study, a total of 199 DEGs (79 up and 120 downregulated genes) were identified in samples from obese children compared with those from lean children within the array dataset GSE29718. The results suggested that MMP9 and ACACB had 
Table II. KEGG and Reactome pathway enrichment analyses for differentially expressed genes $(\mathrm{P}<0.05)$.

A, KEGG pathway

\begin{tabular}{llrl}
\hline ID & \multicolumn{1}{c}{ Description } & No. of genes & P-value \\
\hline $\begin{array}{l}\text { Upregulated } \\
04514\end{array}$ & Cell adhesion molecules & 4 & $1.12 \times 10^{-2}$ \\
04145 & Phagosome & 4 & $1.80 \times 10^{-2}$ \\
00520 & Amino sugar and nucleotide sugar metabolism & 2 & $4.01 \times 10^{-2}$ \\
04670 & Leukocyte transendothelial migration & 3 & $4.11 \times 10^{-2}$ \\
05144 & Malaria & Autoimmune thyroid disease & 2 \\
05320 & & 2 & $4.47 \times 10^{-2}$ \\
Downregulated & Nitrogen metabolism & $4.63 \times 10^{-2}$ \\
00910 & Propanoate metabolism & 3 & \\
00640 & Vascular smooth muscle contraction & 3 & $1.00 \times 10^{-3}$ \\
04270 & Arrhythmogenic right ventricular cardiomyopathy & 4 & $2.60 \times 10^{-3}$ \\
05412 & Alanine, aspartate and glutamate metabolism & 3 & $1.77 \times 10^{-2}$ \\
00250 & Glycerophospholipid metabolism & 2 & $2.60 \times 10^{-2}$ \\
00564 & Dilated cardiomyopathy & 3 & $3.11 \times 10^{-2}$ \\
05414 & & $3.18 \times 10^{-2}$ & $4.28 \times 10^{-2}$ \\
\hline
\end{tabular}

B, Reactome pathway

\begin{tabular}{|c|c|c|c|}
\hline ID & Description & No. of genes & P-value \\
\hline \multicolumn{4}{|c|}{ Upregulated } \\
\hline 168256 & Immune system & 12 & $4.20 \times 10^{-3}$ \\
\hline 1280218 & Adaptive immune system & 7 & $9.10 \times 10^{-3}$ \\
\hline 1474244 & Extracellular matrix organization & 5 & $1.22 \times 10^{-2}$ \\
\hline 2173782 & Binding and uptake of ligands by scavenger receptors & 2 & $2.10 \times 10^{-2}$ \\
\hline 199992 & Trans-golgi network vesicle budding & 2 & $2.41 \times 10^{-2}$ \\
\hline 421837 & Clathrin-derived vesicle budding & 2 & $2.41 \times 10^{-2}$ \\
\hline 168249 & Innate immune system & 7 & $3.70 \times 10^{-2}$ \\
\hline 1433557 & Signaling by SCF-KIT & 3 & $3.82 \times 10^{-2}$ \\
\hline 2219530 & Constitutive signaling by aberrant PI3K in cancer & 2 & $4.32 \times 10^{-2}$ \\
\hline \multicolumn{4}{|c|}{ Downregulated } \\
\hline 5362517 & Signaling by retinoic acid & 4 & $4.00 \times 10^{-4}$ \\
\hline 418457 & cGMP effects & 3 & $9.00 \times 10^{-4}$ \\
\hline 392154 & Nitric oxide stimulates guanylate cyclase & 3 & $9.7 \times 10^{-4}$ \\
\hline 109582 & Hemostasis & 10 & $3.00 \times 10^{-3}$ \\
\hline 204174 & Regulation of pyruvate dehydrogenase complex & 2 & $4.10 \times 10^{-3}$ \\
\hline
\end{tabular}

KEGG, Kyoto Encyclopedia of Genes and Genomes; cGMP, cyclic guanosine monophosphate; PI3K, phosphoinositide-3 kinase; SCF, stem cell factor.

a high degree of interaction in the PPI network and were marker genes in obese children. Moreover, upregulated DEGs were significantly enriched in Reactome pathways of the immune system, and in various associated terms of the GO-BP category with higher P-values. Furthermore, MMP9, an upregulated DEG, was enriched in the term immune system processes of the GO-BP category.

MMPs, a family of zinc-dependent endopeptidases, decrease components of basement membranes and extracellular matrix.
A previous study showed that the MMP9 is upregulated in obese children (32). Florys et al (33) reported increased levels of MMP9 in obese children with type 1 diabetes mellitus. Furthermore, MMP9 has a significant role in the expansion of fat cells and adipose tissue (34). It has been suggested that extracellular matrix remodeled by MMP9 regulated adipocyte differentiation, which then resulted in the progression of obesity (35). Moreover, The $\mathrm{C}^{-1562} \mathrm{~T}$ polymorphism of the MMP9 gene promoter may lead to the development of obesity (35). 


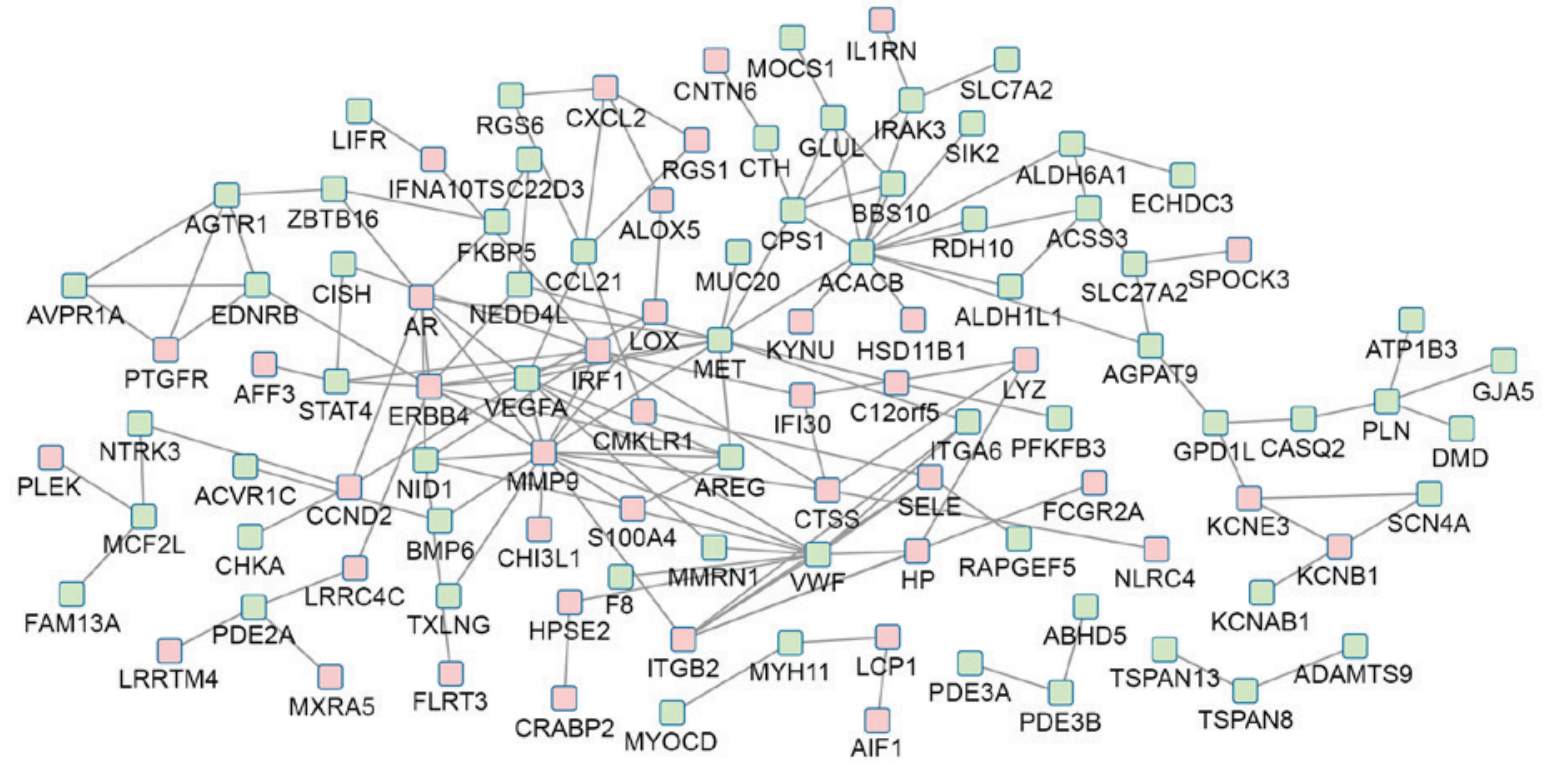

Figure 2. Protein-protein interaction network of differentially expressed genes. Red nodes represent upregulated and green nodes represent downregulated genes.

A
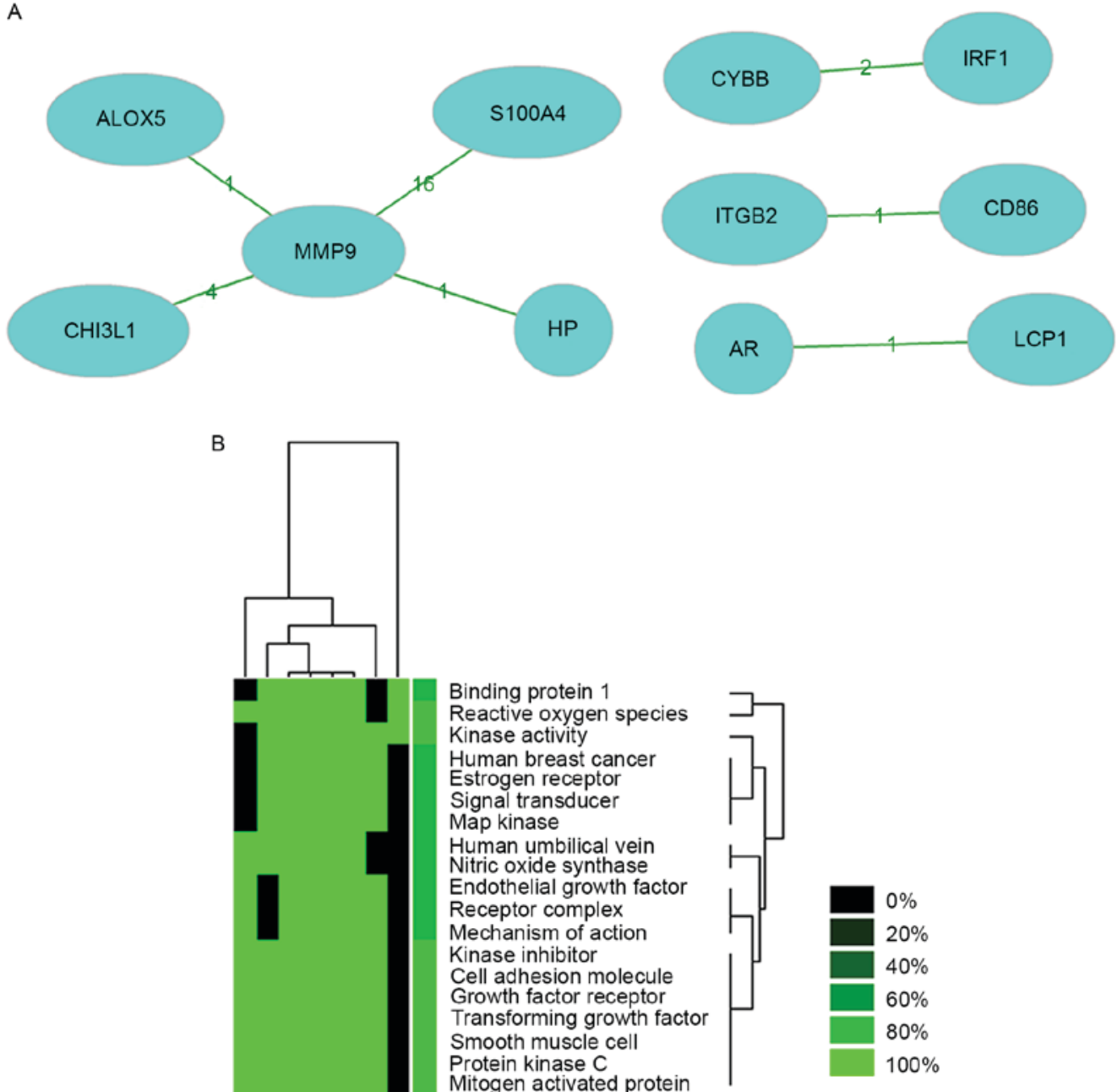

Binding protein 1

Reactive oxygen species

Kinase activity

Human breast cancer

Estrogen receptor

Signal transducer

Map kinase

Human umbilical vein

Nitric oxide synthase

Endothelial growth factor

Receptor complex

Mechanism of action

Kinase inhibitor

Cell adhesion molecule

Growth factor receptor

Transforming growth facto

Smooth muscle cell

Protein kinase $C$

Mitogen activated protein

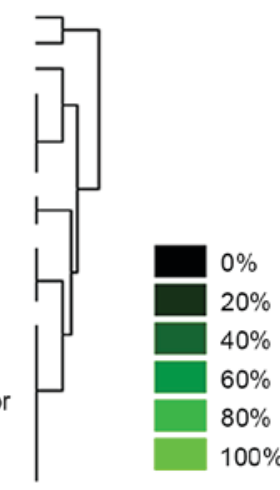

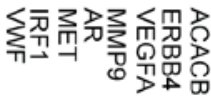

Figure 3. (A) Co-citation network of 8 genes with a higher degree of expression according to previous studies. The numbers on the linking lines indicate the number of studies co-cited. (B) Significant enrichment results of these 8 genes from previous studies. MMP, matrix metalloproteinase; ACACB, acetyl-CoA carboxylase $\beta$; VEGFA, vascular endothelial growth factor $\alpha$; VWF, von Willebrand factor; AR, androgen receptor; IRF, interferon regulatory factor. 


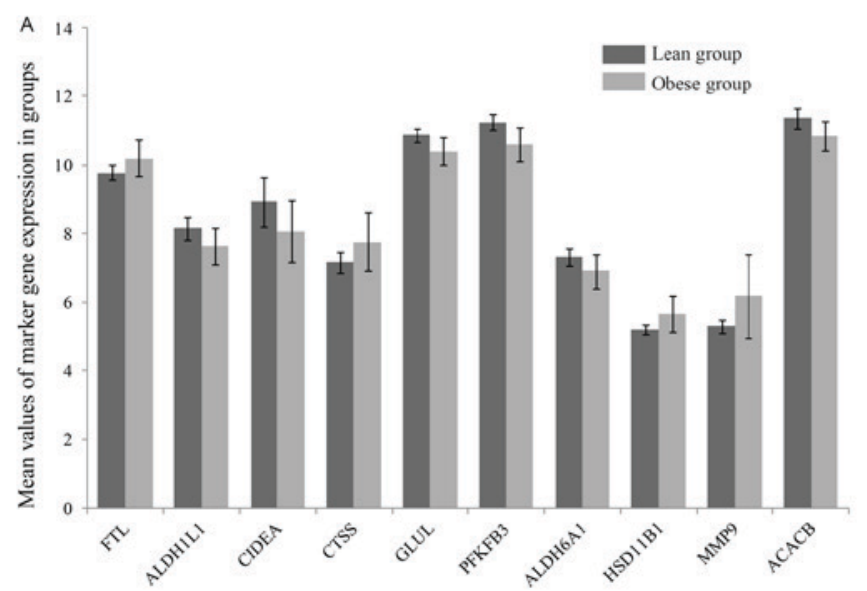

B

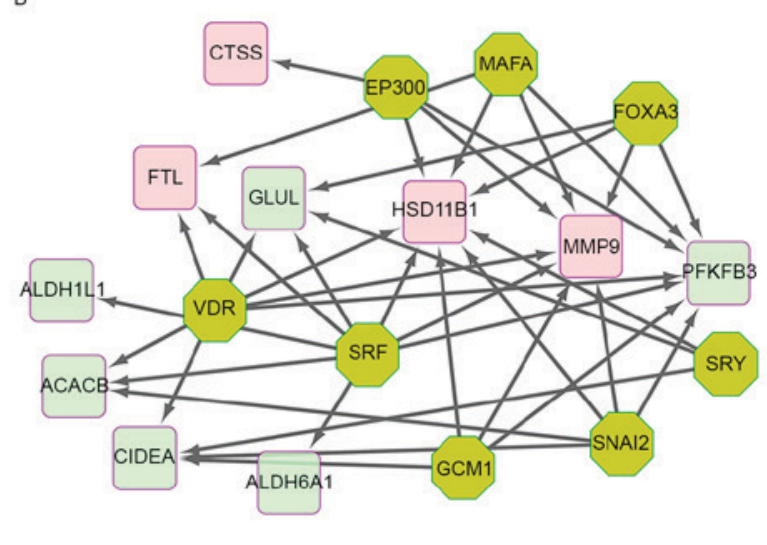

Figure 4. (A) Expression levels of 11 marker genes in samples from obese and lean children which had been listed as obesity-associated marker genes in the comparative toxicogenomics database. (B) Regulatory transcription factor network of the marker genes. MMP, matrix metalloproteinase; ACACB, acetyl-CoA carboxylase $\beta$. Red nodes represent upregulated genes, green nodes represent downregulated genes and yellow nodes represent regulating factors. EP300, E1A binding protein P300; FOXA3, forkhead box A3; SRY, sex-determining region; HSD11B1, hydroxysteroid 11- $\beta$ dehydrogenase 1; ALDH6A1, aldehyde dehydrogenase 6 family member A1; PFKFB3, 6-phosphofructo-2-kinase/fructose-2,6-biphosphatase3; GLUL, glutamate-ammonia ligase; CTSS, cathepsin S; CIDEA, cell death-inducing DFFA-like effector A; FTL, ferritin light chain; VDR, vitamin D receptor; SRF, serum response factor; GCM1, glial cells missing homolog 1; SNAI2, Snail family transcriptional repressor 2.

Table III. Hub genes identified by the present study in the literature.

\begin{tabular}{lcrr}
\hline Gene & Co-genes (n) & Co-citations (n) & Total (n) \\
\hline MMP9 & 4 & 22 & 18,527 \\
ITGB2 & 1 & 1 & 6,736 \\
ALOX5 & 1 & 1 & 325 \\
CD86 & 1 & 1 & 7,281 \\
S100A4 & 1 & 16 & 977 \\
AR & 1 & 1 & 13,054 \\
HP & 1 & 1 & 6,963 \\
LCP1 & 1 & 1 & 289 \\
CHI3L1 & 1 & 4 & 729 \\
CYBB & 1 & 2 & 2,048 \\
IRF1 & 1 & 2 & 1,885 \\
\hline
\end{tabular}

MMP, matrix metalloproteinase; AR, androgen receptor; IRF, interferon regulatory factor; ITGB2, integrin $\beta 2$; ALOX5, arachidonate 5-lipoxygenase; S100A4, S100 calcium binding protein A4; HP, haptoglobin; LCP1, lymphocyte cytosolic protein 1; CHI3L1, chitinase 3-like 1; CYBB, cytochrome B-245 $\beta$ chain. Co-genes indicate the number of linked genes. Co-citations indicate the number of other genes co-cited with this gene from literature mining.

In the present study, MMP9, which had the highest degree of interaction in the PPI network, was identified as a marker gene in obese children. Thus, the results of the present study are in accordance with those of previous ones and suggest that MMP9 may be a key gene associated with child obesity.

Furthermore, ACACB, which also had a high degree of interaction in the PPI network, was identified as a marker gene associated with child obesity in the present study. $\mathrm{ACACB}$, one isoform of $\mathrm{ACAC}$, is involved in fatty acid oxidation $(36,37)$. The variants of the ACACB allele are associated with obesity (38). A previous study showed that body fat may act as a mechanism of metabolic adaptation and a signal that downregulated ACACB expression in adipose tissue (39). In addition, rs2268388 polymorphisms of ACACB are associated with severe obesity and ACACB has a significant part in disorders associated with energy metabolism such as obesity (38). Furthermore, ACACB is involved in triglyceride synthesis and triglyceride contents of soleus muscle were greater in obese than in lean individuals $(40,41)$. Therefore, ACACB may be a key gene associated with obesity.

In addition, upregulated DEGs identified by the present study were found to be significantly enriched in Reactome pathways associated with the immune system. Obesity, malnutrition by excess, is associated with immune dysfunction (42). It has been suggested that the pathogenesis of obesity includes an impaired immune system and cell-mediated immune responses, and altered immune function (43). Besides, adipose tissues of obese individuals showed signs of immune response pathway activation compared with those in lean people in a study on weight-discordant twins (44). A previous study has demonstrated that the cell-mediated immune response is impaired in obese children (42). Tam et al (18) found an over-representation of the immune and inflammatory response in performing gene ontology analyses in adipose tissues of children. In addition, activation of JNK1 and inhibitor of $\kappa \mathrm{B}$ kinase pathways regulate inflammatory and stress mechanisms in obesity, and numerous components of the immune system, such as macrophages, have roles in the pathologies of obesity-associated inflammation (45). The accumulation of macrophages and development of crown-like structures are involved in adipose tissue inflammation in obesity (45). Furthermore, obesity is associated with increased immune cells in the central nervous system (46). Therefore, pathways of the immune system may be significantly associated with child obesity. Besides, MMP9, an upregulated DEG, was enriched in the term immune system processes in the GO-BP category in 
the present study. It is thus speculated that MMP9 may be involved in child obesity via immune system pathways.

The results of the present study showed that MMP9, ACACB and immune system pathways may have important roles in child obesity. Manco et al (47) suggested that massive weight loss had effects on the innate immune system in morbidly obese women. Laimer et al (48) indicated that weight loss in obese women was associated with decreased MMP9. Thus, MMP9 and immune system pathways may also have significant roles in adult obesity. However, to the best of our knowledge, no previous study found that ACACB was directly associated with the development of adult obesity, and thus, this gene may not have any direct relevance in adult obesity. ACACB may be differentially expressed in child obesity compared with adult obesity, which remains to be clarified in future studies. Genes and pathways associated with child and adult obesity as well as their differences are still required to be fully elucidated.

In conclusion, MMP9, ACACB and immune system pathways may have significant roles in child obesity. However, further studies on key genes and pathways associated with child obesity are required. In addition, the molecular mechanisms of adult obesity are required to be elucidated and to be compared with those of child obesity to identify differences between them.

\section{Acknowledgements}

This study was supported by the Special Foundation for Taishan Scholars (grant no. ts20110814) and a project of the Shandong Province Science and Technology Program (grant no. 2014GSF118179).

\section{References}

1. Haslam DW and James WP: Obesity. Lancet 366: 1197-1209, 2005.

2. Hedley AA, Ogden CL, Johnson CL, Carroll MD, Curtin LR and Flegal KM: Prevalence of overweight and obesity among US children, adolescents, and adults, 1999-2002. Jama 291: 2847-2850, 2004.

3. Martorell R, Kettel Khan L, Hughes ML and GrummerStrawn LM: Overweight and obesity in preschool children from developing countries. Int J Obes Relat Metab Disord 24: 959-967, 2000

4. National Institutes of Health: The practical guide: identification, evaluation and treatment of overweight and obesity in adults. National Heart, Lung and Blood Institute, 2002.

5. Freedman DS, Khan LK, Dietz WH, Srinivasan SR and Berenson GS: Relationship of childhood obesity to coronary heart disease risk factors in adulthood: The Bogalusa heart study. Pediatrics 108: 712-718, 2001.

6. Wajchenberg BL: Subcutaneous and visceral adipose tissue: Their relation to the metabolic syndrome. Endocr Rev 21: 697-738, 2000

7. Drincic AT, Armas LA, Diest EE and Heaney RP: Volumetric dilution, rather than sequestration best explains the low vitamin D status of obesity. Obesity (Silver Spring) 20: 1444-1448, 2012.

8. Kim KA, Gu W, Lee IA, Joh EH and Kim DH: High fat diet-induced gut microbiota exacerbates inflammation and obesity in mice via the TLR4 signaling pathway. PLoS One 7: e47713, 2012.

9. Farooqi IS and O'Rahilly S: Mutations in ligands and receptors of the leptin-melanocortin pathway that lead to obesity. Nat Clin Pract Endocrinol Metab 4: 569-577, 2008.

10. Vernia S, Cavanagh-Kyros J, Barrett T, Jung DY, Kim JK and Davis RJ: Diet-induced obesity mediated by the JNK/DIO2 signal transduction pathway. Genes Dev 27: 2345-2355, 2013.
11. Hwang JT, Kim SH, Lee MS, Kim SH, Yang HJ, Kim MJ, Kim HS, Ha J, Kim MS and Kwon DY: Anti-obesity effects of ginsenoside $\mathrm{Rh} 2$ are associated with the activation of AMPK signaling pathway in 3T3-L1 adipocyte. Biochem Biophys Res Commun 364: 1002-1008, 2007.

12. Vohl MC, Sladek R, Robitaille J, Gurd S, Marceau P, Richard D, Hudson TJ and Tchernof A: A survey of genes differentially expressed in subcutaneous and visceral adipose tissue in men. Obes Res 12: 1217-1222, 2004.

13. Linder K, Arner P, Flores-Morales A, Tollet-Egnell P and Norstedt G: Differentially expressed genes in visceral or subcutaneous adipose tissue of obese men and women. J Lipid Res 45: 148-154, 2004.

14. Frayling TM, Timpson NJ, Weedon MN, Zeggini E, Freathy RM, Lindgren CM, Perry JR, Elliott KS, Lango H, Rayner NW, et al: A common variant in the FTO gene is associated with body mass index and predisposes to childhood and adult obesity. Science 316: 889-894, 2007.

15. Dina C, Meyre D, Gallina S, Durand E, Körner A, Jacobson P, Carlsson LM, Kiess W, Vatin V, Lecoeur C, et al: Variation in FTO contributes to childhood obesity and severe adult obesity. Nat Genet 39: 724-726, 2007.

16. Zhang S, Wang B, Shi J and Li J: Network-based association study of obesity and type 2 diabetes with gene expression profiles. Biomed Res Int 2015: 619730, 2015.

17. Chen Y, Li L and Xu R: Disease comorbidity network guides the detection of molecular evidence for the link between colorectal cancer and obesity. AMIA Jt Summits Transl Sci Proc 2015: 201-206, 2015.

18. Tam CS, Heilbronn LK, Henegar C, Wong M, Cowell CT, Cowley MJ, Kaplan W, Clément K and Baur LA: An early inflammatory gene profile in visceral adipose tissue in children. Int J Pediatr Obes 6: e360-e363, 2011.

19. Gautier L, Cope L, Bolstad BM and Irizarry RA: affy-analysis of Affymetrix GeneChip data at the probe level. Bioinformatics 20: 307-315, 2004.

20. Carvalho BS and Irizarry RA: A framework for oligonucleotide microarray preprocessing. Bioinformatics 26: 2363-2367, 2010.

21. Ritchie ME, Phipson B, Wu D, Hu Y,Law CW, Shi W and Smyth GK: limma powers differential expression analyses for RNA-sequencing and microarray studies. Nucleic Acids Res 43: e47, 2015.

22. Smyth GK: Linear models and empirical Bayes methods for assessing differential expression in microarray experiments. Stat Appl Genet Mol Biol 3: Article3, 2004.

23. Ashburner M, Ball CA, Blake JA, Botstein D, Butler H, Cherry JM, Davis AP, Dolinski K, Dwight SS, Eppig JT, et al: Gene Ontology: Tool for the unification of biology. Nat Genet 25: 25-29, 2000.

24. Kanehisa M and Goto S: KEGG: Kyoto encyclopedia of genes and genomes. Nucleic Acids Res 28: 27-30, 2000.

25. Beloqui A, Guazzaroni ME, Pazos F, Vieites JM, Godoy M, Golyshina OV, Chernikova TN, Waliczek A, Silva-Rocha R, Al-Ramahi Y, et al: Reactome array: Forging a link between metabolome and genome. Science 326: 252-257, 2009.

26. Szklarczyk D, Franceschini A, Wyder S, Forslund K, Heller D, Huerta-Cepas J, Simonovic M, Roth A, Santos A, Tsafou KP, et al: STRING v10: Protein-protein interaction networks, integrated over the tree of life. Nucleic Acids Res 43 (Database Issue): D447-D452, 2015.

27. Shannon P, Markiel A, Ozier O, Baliga NS, Wang JT, Ramage D, Amin N, Schwikowski B and Ideker T: Cytoscape: A software environment for integrated models of biomolecular interaction networks. Genome Res 13: 2498-2504, 2003.

28. Wang JH, Zhao LF, Lin P, Su XR, Chen SJ, Huang LQ, Wang HF, Zhang H, Hu ZF, Yao KT and Huang ZX: GenCLiP 2.0: A web server for functional clustering of genes and construction of molecular networks based on free terms. Bioinformatics 30: 2534-2536, 2014.

29. Jensen LJ, Saric J and Bork P: Literature mining for the biologist: From information retrieval to biological discovery. Nat Rev Genet 7: 119-129, 2006.

30. Davis AP, Grondin CJ,Lennon-Hopkins K, Saraceni-Richards C, Sciaky D, King BL, Wiegers TC and Mattingly CJ: The Comparative Toxicogenomics Database's 10th year anniversary: Update 2015. Nucleic Acids Res 43 (Database Issue): D914-D920, 2015.

31. Janky R, Verfaillie A, Imrichová H, Van de Sande B, Standaert L, Christiaens V, Hulselmans G, Herten K, Naval Sanchez M, Potier D, et al: iRegulon: From a gene list to a gene regulatory network using large motif and track collections. PLoS Comput Biol 10: e1003731, 2014. 
32. Głowińska-Olszewska B and Urban M: Elevated matrix metalloproteinase 9 and tissue inhibitor of metalloproteinase 1 in obese children and adolescents. Metabolism 56: 799-805, 2007.

33. Florys B, Głowińska B, Urban $M$ and Peczyńska J: Metalloproteinases MMP-2 and MMP-9 and their inhibitors TIMP-1 and TIMP-2 levels in children and adolescents with type 1 diabetes. Endokrynol Diabetol Chor Przemiany Materii Wieku Rozw 12: 184-189, 2006 (In Polish).

34. Andrade VL, Petruceli E, Belo VA, Andrade-Fernandes CM, Caetano Russi CV, Bosco AA, Tanus-Santos JE and Sandrim VC: Evaluation of plasmatic MMP-8, MMP-9, TIMP-1 and MPO levels in obese and lean women. Clin Biochem 45: 412-415, 2012

35. Andrade VL, Fernandes KS, Bosco AA, Tanus-Santos JE and Sandrim VC: Functional polymorphism located in MMP-9 gene promoter is strongly associated with obesity. DNA Cell Biol 31: 1054-1057, 2012

36. Abu-Elheiga L, Matzuk MM, Abo-Hashema KA and Wakil SJ Continuous fatty acid oxidation and reduced fat storage in mice lacking acetyl-CoA carboxylase 2. Science 291: 2613-2616, 2001.

37. Abu-Elheiga L, Brinkley WR, Zhong L, Chirala SS, Woldegiorgis G and Wakil SJ: The subcellular localization of acetyl-CoA carboxylase 2. Proc Natl Acad Sci USA 97: 1444-1449, 2000.

38. Riancho JA, Vázquez L, García-Pérez M, Sainz J, Olmos JM, Hernández JL, Pérez-López J, Amado JA, Zarrabeitia MT, Cano A and Rodríguez-Rey JC: Association of ACACB polymorphisms with obesity and diabetes. Mol Genet Metab 104: 670-676, 2011

39. Ma L, Mondal AK, Murea M, Sharma NK, Tönjes A, Langberg KA, Das SK, Franks PW, Kovacs P, Antinozzi PA, et al: The effect of ACACB cis-variants on gene expression and metabolic traits. PLoS One 6: e23860, 2011.

40. Wang JC, Gray NE, Kuo T and Harris CA: Regulation of triglyceride metabolism by glucocorticoid receptor. Cell Biosci 2: 19, 2012.
41. Sinha R, Dufour S, Petersen KF, LeBon V, Enoksson S, Ma YZ, Savoye M, Rothman DL, Shulman GI and Caprio S: Assessment of skeletal muscle triglyceride content by (1) H nuclear magnetic resonance spectroscopy in lean and obese adolescents: Relationships to insulin sensitivity, total body fat, and central adiposity. Diabetes 51: 1022-1027, 2002.

42. Marti A, Marcos A and Martinez JA: Obesity and immune function relationships. Obes Rev 2: 131-140, 2001

43. de Heredia FP, Gómez-Martínez S and Marcos A: Obesity, inflammation and the immune system. Proc Nutr Soc 71: 332-338, 2012.

44. Pietiläinen KH, Róg T, Seppänen-Laakso T, Virtue S, Gopalacharyulu P, Tang J, Rodriguez-Cuenca S, Maciejewski A, Naukkarinen J, Ruskeepää AL, et al: Association of lipidome remodeling in the adipocyte membrane with acquired obesity in humans. PLoS Biol 9: e1000623, 2011.

45. Patel PS, Buras ED and Balasubramanyam A: The role of the immune system in obesity and insulin resistance. J Obes 2013: 616193, 2013

46. Buckman LB, Hasty AH, Flaherty DK, Buckman CT, Thompson MM, Matlock BK, Weller K and Ellacott KL: Obesity induced by a high-fat diet is associated with increased immune cell entry into the central nervous system. Brain Behav Immun 35: 33-42, 2014

47. Manco M, Fernandez-Real JM, Equitani F, Vendrell J, Valera Mora ME, Nanni G, Tondolo V, Calvani M, Ricart W, Castagneto $\mathrm{M}$ and Mingrone G: Effect of massive weight loss on inflammatory adipocytokines and the innate immune system in morbidly obese women. J Clin Endocrinol Metab 92: 483-490, 2007.

48. Laimer M, Kaser S, Kranebitter M, Sandhofer A, Mühlmann G, Schwelberger H, Weiss H, Patsch JR and Ebenbichler CF: Effect of pronounced weight loss on the nontraditional cardiovascular risk marker matrix metalloproteinase- 9 in middle-aged morbidly obese women. Int J Obes (Lond) 29: 498-501, 2005. 